\title{
Optical Managing Project — Effectively Survivability and Monitoring System
}

\author{
A. Premadi, A. Effendi, and B. Antonov
}

\begin{abstract}
In this paper we share an experience on managing the Fiber-to-the Home (FTTH) network for next generation customer access network. We ensure our proposed network system able to localizing the failure that occurs at end user by means of our new concept prototype device. Detecting fault on traffic with carried by the same wavelength is an obstacle due to the single photo-detector used but with our proposed configuration we are able to detect the fault and location specifically. Additional, we developed also the prototypes for easiness maintenance for the future implementation. Finally we show excellence results to indicate our proposed system is the better proposal for new generation network with survivability and effective monitoring solution.
\end{abstract}

Index Terms - FTTH, failure, monitoring, restoration.

\section{INTRODUCTION}

Today, optical cable has been used in communication networks to replace copper cable. It has abundant bandwidth for carrying information also over longer distance than copper or coaxial cable. Optical cable is selected due to the advantages it has over other transmission media. It carries information as optical pulses, immune to interference, crosstalk, and noise [1]. FTTH deployments are mostly based on Passive Optical Network (PON) technology. It is an attractive solution for next-generation broadband access networks that deliver converged data, video, and voice over a single optical access system. Multiple services can easily be provided to the end users through PON technology. Due to simple, cost-effective, and scalable features, service providers can deploy the access network infrastructure, such as Fiber to the Home (FTTH) through the use of PONs without impairing their competitive positions. A PON is a point-to-multipoint optical network without any active elements from source to destination and the optical signals are replicated passively by the splitter. The PON architecture basically comprises of an optical line terminal (OLT) residing in the central office (CO), a feeder fiber, a passive optical splitter/combiner, and connects a group of associated optical network units (ONUs) near subscribers, shown in Figure 1. Additionally, the ONU can be deployed in homes as in FTTH, or connected to customers through existing copper wire, such as very high rate Digital Subscriber Line (VDSL) or Cable modem [2], [3]. In the EPON, any data or control information is delivered in

Manuscript received October 4, 2015; revised December 28, 2015. This work was supported in part by the Indonesian Goverment under Grant Hibah Bersaing.

The authors are with the Electrical Engineering Department, Institut Teknologi Padang, and Indonesia (e-mail: aswir.premadi@itp.ac.id, asnal.effendi@itp.ac.id, antonov@itp.ac.id). standard Ethernet frames. The EPON comprises both upstream and downstream data transmissions working on the 1490-nm wavelength for the down direction, the 1310-nm wavelength for the up direction, and the $1550-\mathrm{nm}$ wavelength reserved for future extensions or additional services, such as Cable-TV broadcasts. In the downstream transmission, the OLT is able to broadcast all the control messages and the data frames to all ONUs through the optical splitter. Each ONU discards or accepts the incoming Ethernet frames depending on the destination address field in the frames. In the upstream direction, all ONUs are served by the OLT using a discovery handshake in the Multi-Point Control Protocol (MPCP) and share a common transmission channel toward the OLT by time-division multiple access schemes (TDMA). Only a single ONU may transmit data in one timeslot to avoid signal collisions. After the ONU is registered by the discovery process, the OLT controls the PON and coordinates the transmission window of ONUs with granted GATE messages, which contain the transmission start time and transmission length of the corresponding ONU. The ONU reports its queue status by piggybacking a REPORT message, which can carry one or more queue information sets, with each set comprising up to eight queues in its transmitting period. Then, the OLT calculates the ONU transmission window length using a collision-free timeslots scheduling algorithm. After receiving the GATE message, the 16- $n s$ resolution counter in the ONU can be synchronized to the OLT clock, then, the ONU returns the transmitting time back in a REPORT message to the OLT in order to measure the ONU round-trip delay.The transmission rate on commercialization FTTH technology is already achieved $2.5 \mathrm{Gbps}$ but at system is already upgraded up to $10 \mathrm{Gbps}$ [4].

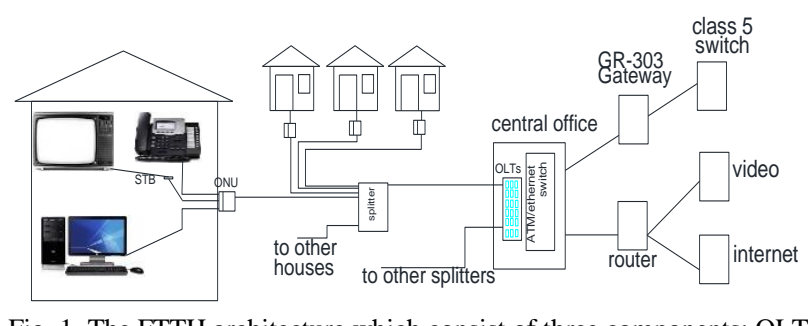

Fig. 1. The FTTH architecture which consist of three components; OLT, ONU and optical splitter.

To avoid signal collisions and to allocate bandwidth fairly in the upstream direction, scheduling algorithms are used, which have been extensively studied. There are two categories of bandwidth allocation schemes on EPONs: fixed bandwidth allocation (FBA) and dynamic bandwidth allocation (DBA).In the FBA scheme, each ONU is assigned fixed timeslots for the data transmission from the ONUs to the OLT at full link capacity. In contrast, DBA improves the 
system by using the bandwidth in a more efficient way; the OLT allocates a variable number of timeslots to each ONU dynamically based on the bandwidth request and ensures the Quality-of-Service (QoS) by a guaranteed service level agreement (SLA) [5], [6].

The PON is commonly deployed as it can offer a cost-efficient and scalable solution to provide huge-capacity optical access [7]. Since the PON can accommodate a large number of subscribers, when any fault occurs in FTTH, the network will without any function behind the break point. Any service outage due to a fiber break can be translated into tremendous financial loss in business for the network service providers [8].

Fault tolerance is also an important issue in PONs. There are two categories of network failures in an EPON, one is a link failure and the other is a node failure [9], [10]. In the link failure case, the failure of the feeder fiber will halt the whole PON system, but a failure of a branch will halt just one ONU. In the node failure case, the failures of OLT or splitter will cause the whole PON system to be on fault. Therefore, the OLT, feeder fiber and splitter are the most critical components in the PON system. Sharing bandwidth to protect the neighboring PONs is an alternative way to reduce the cost of protection [11], [12].

\section{DESIGNING TO IMPROVE NETWORK}

The proposed system architecture is presented in Fig. 2. The proposed system is associated with optical monitoring, remotely controlling, failure detection, and automatic recovery apparatus. The communication system between $\mathrm{CO}$ and end user is done via the wireless network to enable the controlled switch that can be handled manually or automatically. The web-based is designed to interface the system to the user operator [11]. Meanwhile, optical fiber fault detector is installed before the ONU is function to detecting fault occurs in the network. In the case of failure, a signal is send to the protection unit to activate the suitable restoration scheme. Our proposed system can restore the network up to the two cases of failures. Our study is continuing to increase the number of failure restoration events.

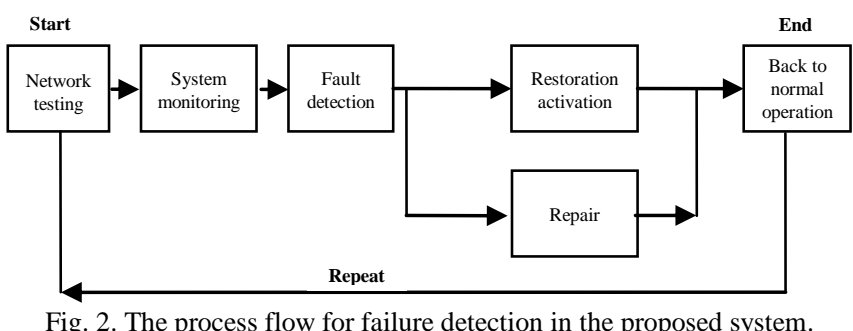

A fault detection method for multiple access communication is proposed. By using the occupancy term of a time-domain equalized signal as a detection system. The optical fiber fault detector is embedded in ONU used to detect any line fault occurs in the network which purposely focused on passive optical network (PON). It is used the tapping mechanism to verify the status of each line by using $10 \mathrm{~dB}$ couplers. With the assisting of optical switch matrix and microcontroller, the status of each line connected to ONU will be identified. The result is the fault number represent occupancy counter which means the number of none traffic flow detected in the specified line during the detection process. In our proposed system, we designed the protection scheme that enable to configure the faults until second order means the failures can occur at two communication lines but the signal can still be transmit to the users.

\section{EXPERIMENTAL SETUP}

Fig. 3 shows the experimental setup to demonstrate the proposed fault monitoring method in FTTH. We used the 20 $\mathrm{km}$ single-mode fiber to connect the OLT with the optical splitter. In this experiment, the wavelength of $1490 \mathrm{~nm}$ is allocated for downstream channel from an OLT and the 1310 $\mathrm{nm}$ is for upstream data service from each ONU. At the OLT, the upstream data signal assign to each ONU separated by the wavelength selective coupler (WSC) were received by the pin receiver. In case of an upstream transmitter failure, the pin receiver cannot receive the data signal, but the monitoring unit can detect the power of the monitoring signal without intermission. If a drop region fiber cut occurs, the pin receiver and the monitoring unit cannot receive the data signal and detect the optical power, respectively.

The monitoring configuration composes of on OTDR, an optical coupler, a $1 \times 8$ optical splitter and optical detector as the sensor. In our experiments, the total insertion lost is nearly $8 \mathrm{db}$. Then, the numbers of ONU are equal to the number of the optical detectors used. In the fiber fault detection, any failure occurs in this region will be sensed by $\mathrm{CO}$ by the $8 \%$ tapped signal through the opticalfiber fault detector that is connected to every access line. The activation signal is then sent to active the dedicated protection scheme. But if fault is still not restored, the shared protection scheme will be activated. The monitoring signal section is responsible for sensing fault and its location whereas generation of activation of signal is sent by activation section in CO.

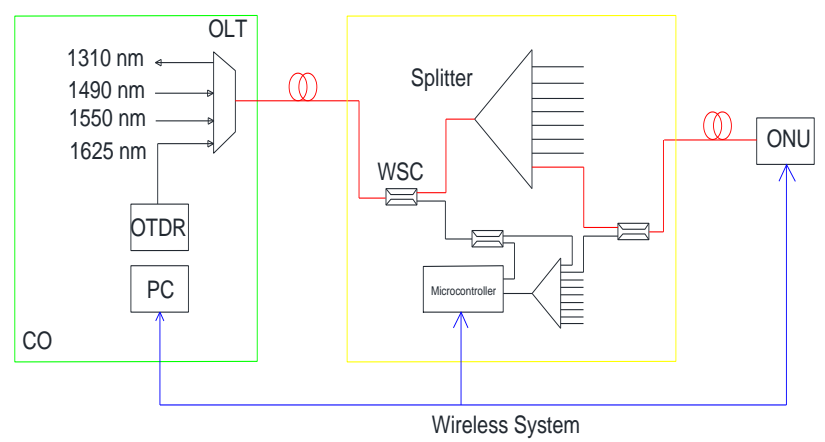

Fig. 3. Experimental setup optical fiber fault on FTTH.

\section{RESULTS AND DisCUSSION}

We used the opti system software to test the proposed FTTH scheme model. The failure conditions are divided into several different fault orders. The first scenario is in first order failure (Condition A) occurs in fiber line, and second condition is in second order failure (Condition $\mid \mathrm{B}$ ) in sequence line was also observed. Fig. 6 shows the eye diagram for 
downstream wavelength. Failure in first order point gives clearer opening of eye diagram rather than condition failure in second order failure. In twice order failure the highest value of dynamic range is achieved since the restoration route mechanism uses 10 numbers of optical switches to perform the proposed scheme to the network. Our results were obtained by observing bit error rates, eye diagrams, optical power levels and dispersion levels. For every number of optical switches, the insertion loss is considered equal to 1.4 $\mathrm{dB}$. Therefore, the measured downstream and upstream dynamic range in $\mathrm{dB}$ in five conditions which is in condition A and condition $B$ are $27.5 \mathrm{~dB}$ and $27.9 \mathrm{~dB}$ respectively.

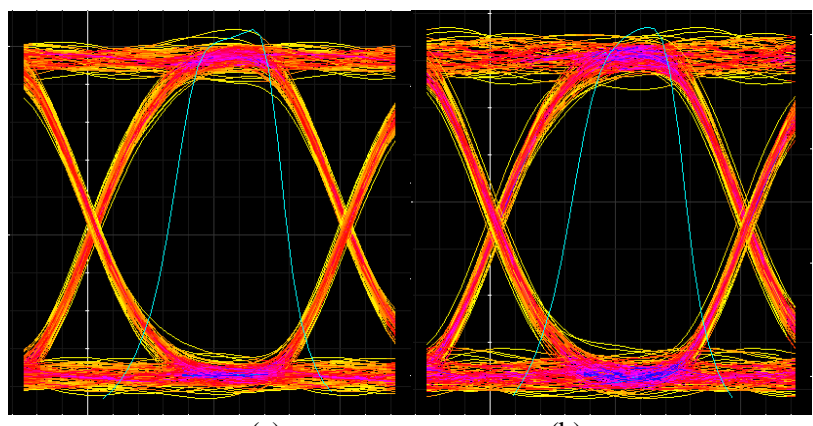

(a)

(b)

Fig. 4. Eye diagram produced from the simulation for (a) condition A and (b) Condition B.

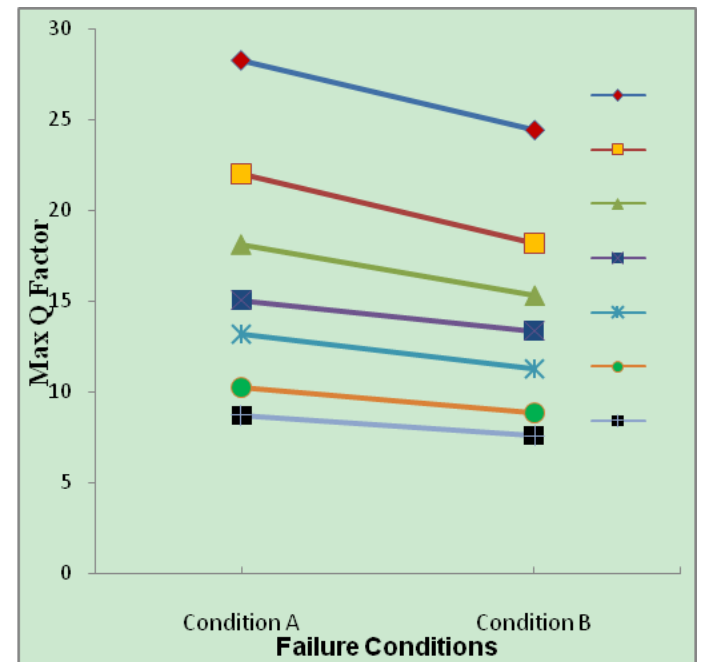

Fig. 5. Max Q Factor achieved from simulation in different fiber length (km) for every condition.

Fig. 5 shows the Max Q Factor performance measured in various transmissions using simulation by sending a long sequence of pseudorandom bits (typical sequence length $2^{15}-1$ ) over a single mode fiber and then detecting it by using a pin receiver. In our design, the maximum fiber length that can be implemented is about $20 \mathrm{~km}$ so that the power receiver at 33.7 $\mathrm{dBm}$ can achieved for Max Q Factor as equal to 6. A higher fiber length represents a smaller value of Max Q Factor in all failure scenarios. The failure condition is varied from condition A to Condition B at $1.25 \mathrm{Gbps}$ with $0 \mathrm{dBm}$ input power. The simulation results are being characterized through BER against fiber length in $\mathrm{km}$. The fiber length is varied from $15 \mathrm{~km}$ to $35 \mathrm{~km}$ in this simulation. However, in order to implement $35 \mathrm{~km}$ fiber length the least minimum Q factor value can only be achieved in normal condition, first order failure and second order failure. The maximum fiber length from OLT to ONU can be used in our design is $20 \mathrm{~km}$ so that the protection scheme for up to forth order failure can be implemented.

According to two failure conditions which is condition A and condition $\mathrm{B}$, the protection route will involve in four, six, eight and ten numbers of optical switch respectively. Figure 6 represents the value of dynamic range in $\mathrm{dBm}$ for every type of failure condition. From the result achieved, the proposed architecture design in all condition can only been used effectively in $-34 \mathrm{dBm}$ sensitivity since the maximum dynamic was achieved from condition $B$ in $33.7 \mathrm{dBm}$. In optical receivers, a receiver is said to be more sensitive if it achieves the same performance with less optical power incident on it The performance criterion for digital receivers is governed by bit-error rate (BER), which is defined as probability of incorrect identification of a bit by the decision circuit of the receiver. A commonly used criterion for digital receivers requires the BER to be below $1 \times 10^{-9}$ (Max Q Factor $\approx 6$ ).

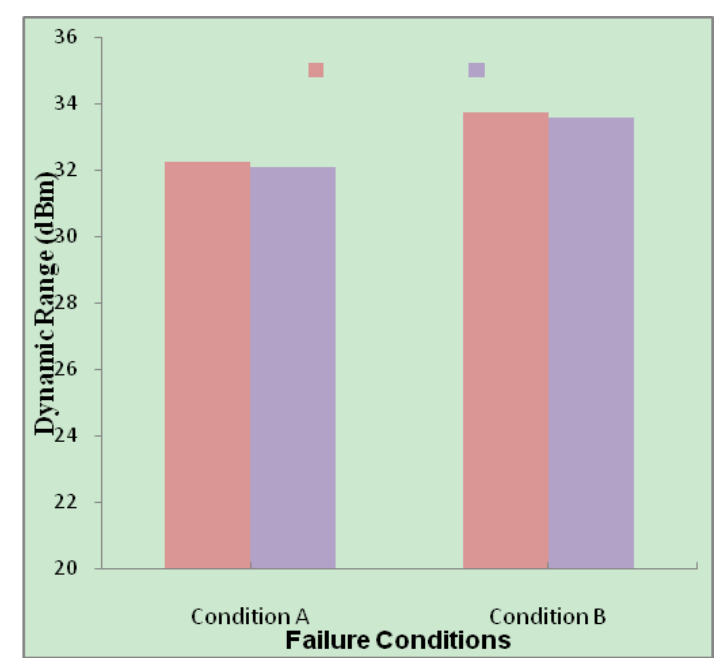

Fig. 6. Dynamic range $(\mathrm{dBm})$ performance of downstream and upstream traffics at two failure conditions observed.

\section{CONCLUSION}

This paper concludes that current optical monitoring technologies are not sufficient for the next generation all optical switched network. For the fault monitoring, the broadband light source and optical switch were required at the OLT and each ONU, respectively. The FTTH employing the proposal monitoring method could detect the link fault in real time.

\section{ACKNOWLEDGMENT}

We would like to thank Prof. Dr. Mohammad Syuhaimi Ab Rahmanfor their valuable suggestion. This work was supported by Dikti under the grant Hibah Bersaing.

\section{REFERENCES}

[1] Conniq.com. (2015). Internet access guide: Fiber-to-the home [Online]. http://www.conniq.com/InternetAccess_FTTH.htm Available:

[2] T. Koonen, "Fiber to the home/fiber to the premises: What, where, and when?" in Proc. the IEEE, vol. 94, no. 5, May 2006.

[3] F. Effenberger and T. S. El-Bawab, "Passive optical networks (PONs): Past, present, and future," Optical Switching and Networking, vol. 6, issue 3, pp. 143-150, 2009

[4] H. He, Trends in Communication Technologies and Engineering Science, Springer Science \& Business Media, 2009. 
[5] M. P. McGarry, M. Maier, and M. Reisslein, "Ethernet PONs: A survey of dynamic bandwidth allocation (DBA) algorithms," IEEE Communication Magazine, vol. 42, pp. 8-15, 2004.

[6] D. Grossman, "New terminology and clarification for diffserv," IETF RFC, 2002.

[7] C. K. Chan, F. Tong, L. K. Chen, K. P. Ho, and D. Lim, "Fiber-fault identification for branched access networks using a wavelength-sweeping monitoring source," IEEE Photon. Technol. Lett., vol. 11, no. 5, 1999, pp. 614-616.

[8] A. A. A. Bakar, M. Z. Jamaludin, F. Abdullah, M. H. Yaacob, M. A. Mahdi, and M. K. Abdullah, "A new technique of real-time monitoring of fiber optic cable networks transmission," Optics and Lasers in Engineering, vol. 45, 2007, pp. 126-130.

[9] I. S. Hwang, Z. D. Shyu, and L. Y. Ke, "A novel fault-tolerant multi-EPON system with sharing protection through bridge ONUs," Photonic Network Communication, vol. 18, pp. 24-38, 2009.

[10] C. Kazmierski, "New colorless 10Gbps remote modulator for multiwavelength access," SPIE Newsroom, 2008.

[11] K. S. Kim, "On the evolution of PON-based FTTH solutions," An International Journal Informatics and Computer Science, vol. 149, pp. 21-30, 2003

[12] A. Premadi, B. C. Ng, M. S. A. Rahman, and K. Jumari, "Access network survivability: an architecture approach for monitoring, protection and restoration in FTTH application," Annals of Telecommunications, vol. 65, issue 5, pp. 263-269, 2010.

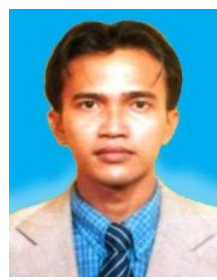

Aswir Premadi received the M.S degree in electrical electronic and system engineering from Universiti Kebangsaan Malayasia in 2011. Currently, he is a lecturer in the Department of Electrical Engineering, Institut Teknologi Padang, Indonesia. He is a member of IEEE. His current research topics include control systems, optical communications and wireless network.

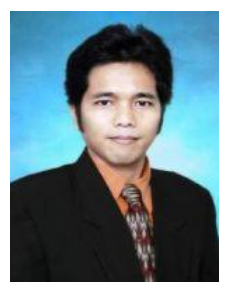

Aswir Premadi received the M.S degree in electrical electronic and system engineering from Universiti Kebangsaan Malayasia in 2011. Currently, he is a lecturer in the Department of Electrical Engineering, Institut Teknologi Padang, Indonesia. $\mathrm{He}$ is a member of IEEE. His current research topics include control systems, optical communications and wireless network.

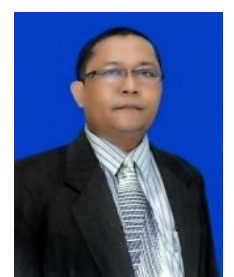

Antovov Bachtiar received M.S degree in electrical electronic and system engineering from Universitas Gadjah Mada in 2005. Currently, he is a lecturer in the Department of Electrical Engineering, Institut Teknologi Padang, Indonesia. His current research topics include power engineering and network management and protection. 